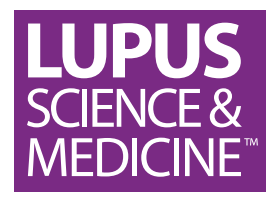

\title{
Vaginal microbiota affects urinary tract infection risk in women with systemic lupus erythematosus: a pilot cross- sectional study from Thailand
}

\author{
Porpon Rotjanapan (D) , ${ }^{1}$ Aunyakant Jaroensukrungruang, ${ }^{1}$ Prapaporn Pisitkun, ${ }^{2}$ \\ Pintip Ngamjanyaporn (D) ,2 Jittima Manonai, ${ }^{3}$ Vorthon Sawaswong, ${ }^{4,5}$ \\ Prangwalai Chanchaem, ${ }^{5,6}$ Sunchai Payungporn ${ }^{5,6}$
}

To cite: Rotjanapan $P$, Jaroensukrungruang $A$, Pisitkun $\mathrm{P}$, et al. Vaginal microbiota affects urinary tract infection risk in women with systemic lupus erythematosus: a pilot crosssectional study from Thailand. Lupus Science \& Medicine 2021;8:e000551. doi:10.1136/ lupus-2021-000551

- Additional supplemental material is published online only. To view, please visit the journal online (http://dx.doi.org/10. 1136/lupus-2021-000551).

Received 5 August 2021 Accepted 14 October 2021

Check for updates

(c) Author(s) (or their employer(s)) 2021. Re-use permitted under CC BY-NC. No commercial re-use. See rights and permissions. Published by BMJ.

For numbered affiliations see end of article.

Correspondence to

Dr Porpon Rotjanapan; porpon rot@mahidol.ac.th

\section{ABSTRACT}

Objective The risk factors associated with urinary tract infections (UTIs) in patients with SLE remain uncertain. We evaluated the vaginal microbiota pattern and its potential UTI-associated risk factors.

Methods A pilot cross-sectional study of patients with SLE was conducted at Ramathibodi Hospital, Bangkok, Thailand, during 2019-2020. Patients' demographic data and relevant information were collected. Vaginal microbiota was assessed in all patients and in 10 healthy volunteers.

Results Fifty-two patients were enrolled (mean age: 46.1 years). All patients had SLE that was in low disease activity. As per the Simpson_e index, the within-group alpha diversity of the vaginal microbiota was low in the SLE with UTI and SLE receiving trimethoprimsulfamethoxazole (TMP-SMX) prophylaxis groups. Multivariate logistic regression analysis revealed that TMP-SMX prophylaxis (adjusted OR (AOR), 30.96; 95\% Cl 3.63 to 264.11; $p=0.002$ ), elevated C3 levels (AOR, $35.33 ; 95 \% \mathrm{Cl} 1.33$ to $936.67 ; p=0.033$ ) and presence of Veillonella dispar in the vaginal microbiota (AOR, 6.68; $95 \% \mathrm{Cl} 1.27$ to $35.07 ; p=0.025$ ) were associated with UTI. Conclusions The vaginal microbiota diversity differed between patients with lupus with and without UTI, and unnecessary administration of TMP-SMX prophylaxis may affect the alpha diversity of the vaginal microbiota.

\section{INTRODUCTION}

SLE is a chronic autoimmune disease involving multiple organ systems. ${ }^{1}$ Infections can trigger the body's immune response, which aggravate the SLE disease activity and result in morbidity and mortality in these patients. ${ }^{2}$ Urinary tract infections (UTIs) are among the most common infections in patients with SLE and have a $36 \%$ prevalence compared with an approximately $12 \%$ prevalence among the general population. ${ }^{34}$

Several risk factors can lead to UTI development, including defects in the host immune response either secondary

\section{Key messages}

What is already known about this subject?

- Urinary tract infections (UTIs) in patients with SLE remain a critical infectious complication that increases patients' morbidity and mortality.

- Comprehensive potential risk factors and vaginal microbiota patterns associated with UTI are unreported in this population.

What does this study add?

- Vaginal microbiota diversity differs between patients with SLE with UTI and those without UTI.

- Unnecessary use of trimethoprim-sulfamethoxazole prophylaxis may affect the alpha diversity of the vaginal microbiota.

How might this impact on clinical practice or future developments?

- This study reflects unnecessary treatment and provides evidence to support discontinuing trimethoprim-sulfamethoxazole prophylaxis in patients with SLE who are receiving low-level immunosuppressive agents.

to immunosuppressive drugs or patients medical conditions; uncontrolled diabetes; anatomical abnormalities of the genitourinary system; imbalances of specific cytokines, such as interleukin $1 \beta$ and interleukin $1 \beta$ antagonists, which are reported to play roles in controlling Escherichia coli colonisation in the genitourinary tract; and altered vaginal microbiota. ${ }^{5-7}$ Kirjavainen $e t a l^{8}$ found that the vaginal microbiota in UTI-prone women exhibited diminished Lactobacillus compositions compared with those in the control group $(p=0.003)$.

One of the first large-scale, non-culturedependent studies of vaginal microbial communities assessed approximately 400 asymptomatic North American women and revealed that most vaginal microbial flora 
(73\%) were dominated by one or more species of Lactobacillus. ${ }^{9}$ Proposed mechanisms by which Lactobacillus may prevent UTI include preventing E. coli from colonising the vagina via competitive mechanisms in which Lactobacillus spp adhere to uroepithelial cells, lowering the vaginal $\mathrm{pH}$ by producing lactic acid, and producing bacteriocins, surfactants and hydrogen peroxide. ${ }^{10}$ Lactic acid concentrations are high in a Lactobacillus-dominated vaginal microbiota, and these conditions produce a potently antimicrobial environment in vitro. ${ }^{11}$

Many factors affect the vaginal microbiota, including a natural or treatment-associated lack of oestrogen, especially during menopause, receiving antimicrobial treatment or contraceptive pills, and having bacterial vaginosis, especially when lacking Lactobacillus spp. ${ }^{10}$ Women lacking vaginal Lactobacillus are at an increased risk of various urogenital disease conditions, including bacterial vaginosis, HIV infection, Neisseria gonorrhoeae infections and vaginal colonisation by E. coli. ${ }^{12}{ }^{13}$

Nielsen $e t \mathrm{al}^{14}$ compared the $E$. coli faecal flora characteristics in patients with UTI with those of healthy controls who had never had a UTI and discovered that $87 \%$ of patients with UTI carried the infecting strain in their faecal flora. Additionally, faecal clones causing the UTI were more often dominant in the faecal flora. These findings support the UTI pathogenesis in which the gut microbiota colonising the vaginal introitus and periurethra may cause an ascending infection via the urethra to the bladder and sometimes the kidneys. ${ }^{10}$ Thus, many patients with SLE may be at risk of UTI after receiving immunosuppressive therapy, treatments affecting normal ovarian function, or antibiotics or if they have altered vaginal microbiota patterns either from impaired ovarian function or imbalanced glucose homeostasis. However, knowledge is lacking regarding patients with SLE and the associated risk factors for UTI considering that these individuals are immunocompromised. Moreover, the vaginal microbiota compositions in women with UTI-associated SLE are unreported. Here, we assessed the associated risk factors for UTI in this population.

\section{Objectives}

The primary objective of this work was to study the potential characteristics of the vaginal microbiota associated with UTI in women with SLE. The secondary objectives were to evaluate the associated risk factors for UTI in women with SLE and explore whether SLE-related treatment affects UTI development in this population.

\section{METHODS}

\section{Study design}

A pilot cross-sectional study of patients with SLE was conducted at Ramathibodi Hospital, a university hospital in Bangkok, Thailand. Women with SLE, according to the Systemic Lupus International Collaborating Clinics 2012 classification criteria, aged $>18$ years visiting the rheumatology or infectious disease clinics from March 2019 to
March 2020 were screened for enrolment regardless of their SLE disease activity, age and immunosuppressive therapy.

Patients were excluded if they had at least one of the following conditions at screening:

1. History of antibiotic use within 2 weeks before enrolment for any reason other than a UTI or suspected UTI.

2. Receipt of hormone replacement therapy, oral contraceptive pills or an intrauterine device.

3. History of vaginal douching within 4 weeks before enrolment.

4. Diagnoses of gynaecological infections within 1 month before enrolment.

5. Known or newly diagnosed non-infectious renal conditions resulting in an abnormal urinalysis at baseline (eg, IgA nephropathy, lupus nephritis).

6 . Need for routine urinary catheterisation or indwelling urinary catheter.

7. Pregnancy.

8. No gynaecological examination was performed for any reason.

9. Diagnoses of any malignancies.

10. Known abnormal genitourinary tract structure.

\section{Definitions}

Urinary tract infection

Diagnosis of UTI was adapted from the Infectious Diseases Society of America and the European Society for Microbiology and Infectious Diseases using the following criteria $^{1516}$ :

1. Relevant clinical presentations: participants with acute dysuria alone or with fever $\left(>37.9^{\circ} \mathrm{C}\right.$ or $1.5^{\circ} \mathrm{C}$ increase above baseline temperature) plus at least one symptom or new or worsening symptoms associated with urination, including urgency, frequency, suprapubic pain, gross haematuria, costovertebral tenderness and/or urinary incontinence.

2. Laboratory diagnostic criteria:

2.1. Presence of $>10$ white cell count per $10^{9} / \mathrm{L} /$ highpower field on urinalysis.

2.2. Evidence of bacteriuria ( $\geq 10^{5}$ colony-forming units $(\mathrm{CFU}) / \mathrm{mL}$ in self-voided urine or $\geq 10^{2} \mathrm{CFU} / \mathrm{mL}$ in single catheterised urine).

Culture-confirmed UTI was defined as those from individuals who met criteria 1, 2.1 and 2.2.

Probable UTI was defined as those from individuals who met only criteria 1 and 2.1 .

High-level immunosuppression

High-level immunosuppression was defined as at least one of the following ${ }^{17}$ :

1. Receipt of corticosteroid therapy of $\geq 20 \mathrm{mg} /$ day of prednisolone or equivalent for $\geq 14$ days.

2. Receipt of methotrexate (MTX) $>0.4 \mathrm{mg} / \mathrm{kg} /$ week or azathioprine $>2 \mathrm{mg} / \mathrm{kg} /$ day or 6-mercaptopurine (6MP) $>1.5 \mathrm{mg} / \mathrm{kg} /$ day. 
3. Receipt of biological immune modulators, including tumour necrosis factor-alpha blockers and rituximab.

4. Receipt of mycophenolate mofetil $>1.5 \mathrm{~g} /$ day or Myfortic $>1080 \mathrm{mg} /$ day.

5. Receipt of cyclophosphamide.

6 . Receipt of a combination of three of more immunosuppressive agents, including prednisolone, MTX, azathioprine, 6-MP, mycophenolate mofetil and Myfortic, at any doses.

\section{Low-level immunosuppression}

Low-level immunosuppression was defined as at least one of the following ${ }^{17}$ :

1. Receipt of systemic corticosteroid therapy of $<20 \mathrm{mg} /$ day of prednisolone or equivalent for $\leq 14$ days or receiving alternate-day corticosteroid therapy.

2. Receipt of MTX $\leq 0.4 \mathrm{mg} / \mathrm{kg} /$ week, azathioprine $\leq 2 \mathrm{mg} / \mathrm{kg} /$ day or $6-\mathrm{MP} \leq 1.5 \mathrm{mg} / \mathrm{kg} /$ day.

3. Receipt of mycophenolate mofetil $<1.5 \mathrm{~g} /$ day or Myfortic $<1080 \mathrm{mg} /$ day.

4. Receipt of a combination of two immunosuppressive drugs, including MTX, azathioprine, mycophenolate mofetil and Myfortic, or combined prednisolone with one immunosuppressive drug including MTX, azathioprine, 6-MP, mycophenolate mofetil and Myfortic.

\section{Active SLE}

Active SLE was defined as SLE in patients with a modified Systemic Lupus Erythematosus Disease Activity Index 2000 (SLEDAI-2K) score $\geq 4$. . $^{19} 20$

\section{Alpha diversity}

Alpha diversity represents bacterial richness, evenness or both within a single sample. ${ }^{21}$

\section{Simpson_e index}

Simpson_e index is one of the most commonly used alpha diversity measures. ${ }^{22}$

\section{Data and sample collection}

Patients' demographic data, menstrual status, cause of menopause, vaginal $\mathrm{pH}$, coexisting medical conditions, modified SLEDAI-2K scores, use of trimethoprimsulfamethoxazole (TMP-SMX) and/or acyclovir as prophylactic agents for opportunistic infections, complete blood count, glycosylated haemoglobin (HbA1C), and fasting plasma glucose, C3 and C4 levels were collected at enrolment. The immunosuppressive drug type and data on the 3-month cumulative dose of each drug were recorded.

On the day of enrolment, vaginal swabs were taken at the posterior fornix to assess the vaginal microbiota and urinalyses were performed on all participants. Five healthy menopausal and five healthy non-menopausal women agreed to participate in the study and were used as controls to compare with the enrolled study population on their menstrual status. All healthy controls were screened for the same inclusion/exclusion criteria as were the patients with SLE. The vaginal swabs were stored at $-80^{\circ} \mathrm{C}$ until DNA extraction. Blank swabs and blank buffer controls were also included.

\section{Sample processing and DNA extraction}

Total DNA was extracted using the GenUP gDNA Extraction Kit (Biotechrabbit, Berlin, Germany) with additional mechanical lysis steps. First, $1 \mathrm{~mL}$ of swab suspension was transferred to a $2 \mathrm{~mL}$ lysis tube and centrifuged at 2000 revolutions per minute (rpm) for $5 \mathrm{~min}$ to pellet the bacterial cells. After removing the supernatant, the sample was added to $200 \mu \mathrm{L}$ of lysis buffer from the extraction kit and lysed using a TissueLyser LT with a $5 \mathrm{~mm}$ stainless steel bead (Qiagen, Hilden, Germany) at $50 \mathrm{~Hz}$ for $5 \mathrm{~min}$. The unlysed residue was pelleted again by centrifuging at $12000 \mathrm{rpm}$ for $2 \mathrm{~min}$. The lysate was then processed following the standard protocol from the extraction kit.

\section{S rDNA amplification}

The amplification method for next-generation sequencing was modified from a previous publication. ${ }^{23}$ Briefly, the V4 region of the bacterial 16S rDNA was amplified using four phasing primer sets (spacers 1-4) containing the target primer sequences 515F: 5'-GTGCCAGCMGCCGCGGTAA-3' and 806R: 5'-GGACTACHVGGGTWTCTAAT-3'. Each $20 \mu \mathrm{L}$ PCR consisted of $20 \mathrm{ng}$ of DNA template, $0.2 \mu \mathrm{M}$ of each primer, $0.2 \mathrm{mM}$ of deoxynucleoside triphosphate, $1 \times$ Phusion Green HF Buffer and $0.4 \mathrm{U}$ of Phusion DNA Polymerase $(2 \mathrm{U} / \mu \mathrm{L})$ (Thermo Scientific, Waltham, Massachusetts, USA). The thermal profile included the following:

1. Initial denaturation at $98^{\circ} \mathrm{C}$ for $30 \mathrm{~s}$.

2. Twenty-five cycles of amplification $\left(98^{\circ} \mathrm{C}\right.$ for $10 \mathrm{~s}, 53^{\circ} \mathrm{C}$ for $25 \mathrm{~s}, 72^{\circ} \mathrm{C}$ for $25 \mathrm{~s}$ ).

A final extension at $72^{\circ} \mathrm{C}$ for $10 \mathrm{~min}$ was done.

\section{Library preparation and next-generation sequencing}

The PCR product was reamplified using another set of primers composed of a dual multiplexing index set and Illumina adapters. The PCR reaction was prepared using the same protocol as the first PCR in a total volume of $40 \mu \mathrm{L}$. For the second PCR, DNA was amplified under the following conditions: $98^{\circ} \mathrm{C}$ for $30 \mathrm{~s}, 25$ amplification cycles $\left(98^{\circ} \mathrm{C}\right.$ for $10 \mathrm{~s}, 60^{\circ} \mathrm{C}$ for $30 \mathrm{~s}, 72^{\circ} \mathrm{C}$ for $\left.30 \mathrm{~s}\right)$ and a final extension at $72^{\circ} \mathrm{C}$ for $10 \mathrm{~min}$. The PCR product was examined using $2 \%$ agarose gel electrophoresis. The expected band ( $440 \mathrm{bp}$ ) was purified using the QIAquick Gel Extraction Kit (Qiagen, Germany). Purified DNA was quantified via quantitative PCR using KAPA Library Quantification Kits for Illumina platforms (Kapa Biosystems, USA). The DNA libraries were pooled at equal amounts. The pooled library was spiked with $20 \%$ PhiX and subsequently pair-end sequenced at $2 \times 250 \mathrm{bp}$ using a MiSeq V.2 reagent kit on the MiSeq sequencing platform (Illumina, San Diego, California, USA).

\section{Data analysis}

Raw data were demultiplexed using MiSeq Reporter Software (V.2.6.2.3). Paired-end FASTQ sequences were analysed using the QIIME2 pipeline (V.2018.8).$^{24}$ Briefly, 
paired-end reads were merged and trimmed based on quality score $(<\mathrm{Q} 30)$. Joined reads were deduplicated and clustered with $97 \%$ similarity using VSEARCH. ${ }^{25}$ Chimeric sequences were also filtered out by the UCHIME algorithm. ${ }^{26}$ The filtered reads were classified based on $99 \%$ operational taxonomic units clustered in the 16S Greengene database (V.2013.8) ${ }^{27}$ using the VSEARCH algorithm.

\section{Statistical analyses}

Categorical variables are presented as frequencies and percentages. Continuous variables are presented as mean and SD or median and IQR for data with and without a normal distribution, respectively. Characteristics between patients with and without UTI were compared via $\chi^{2}$ or Fisher's exact tests for categorical variables and MannWhitney U test or Student's t-test for continuous variables. ORs and their 95\% CIs were calculated. Two-tailed tests were used to determine statistical significance, and $\mathrm{p}<0.05$ was considered significant. All variables with $\mathrm{p}<0.05$ in the univariate analysis were considered for inclusion in the multivariate analysis. All statistical analyses were performed using SPSS V.17.0 statistical software.

\section{RESULTS}

\section{Baseline characteristics}

Among 1500 patients with SLE in the rheumatology clinic, 104 female patients were screened for enrolment and 52 patients agreed to participate in the study; 18 $(34.6 \%)$ were diagnosed with UTI (17 with probable UTI and one with confirmed UTI). The mean age of all patients was $46.1 \pm 12.7$ years, and 33 of $52(63.5 \%)$ were in menopause. Among those in menopause, 29 $(87.9 \%)$ were undergoing natural menopause (table 1$)$. The proportion of menopausal patients was higher in the SLE without UTI group (24 of 34 patients, 70.6\%) than in the SLE with UTI group (9 of 18 patients, $50 \%$; $\mathrm{p}=0.226)$. Of the 52 patients, $23(44.2 \%)$ had coexisting conditions: 9 (17.3\%) had hypertension, $5(9.6 \%)$ had hyperlipidaemia, $4(7.7 \%)$ had well-controlled diabetes (mean HbA1C of $5.85 \% \pm 0.87 \%$ ), $3(5.8 \%)$ had myoma uteri, $1(1.9 \%)$ had undergone kidney transplantation and $1(1.9 \%)$ had HIV. No patients in the SLE without UTI group had myoma uteri, whereas 3 of 18 (16.7\%) in the SLE with UTI group had myoma uteri $(\mathrm{p}=0.037)$. All patients were in remission or low disease activity with a median modified SLEDAI-2K of 0 (range, $0-1$ ). None of these developed active SLE necessitating treatment modification during the study period. The common immunosuppressive drug prescribed was prednisolone (44 of 52 patients, $84.6 \%$; table 1 ). The drug type and 3-month cumulative immunosuppressive drug doses did not differ between the SLE with UTI and SLE without UTI groups (tables 1 and 2). Of 52 patients, $11(21.2 \%)$ received TMPSMX as a prophylactic agent, whereas $18(34.6 \%)$ patients received acyclovir for prophylaxis. Nine of 18 (50\%) patients receiving TMP-SMX prophylaxis developed UTI compared with 2 of 34 (5.9\%) patients in the SLE without UTI group $(\mathrm{p}<0.001)$. Baseline laboratory values did not differ between the groups, as shown in online supplemental data, except for the UTI group which had a higher mean C3 level $(1.1 \pm 0.2 \mathrm{~g} / \mathrm{L}$ vs $0.9 \pm 0.3 \mathrm{~g} / \mathrm{L} ; \mathrm{p}=0.027)$. The mean age among healthy menopausal individuals was $61 \pm 6.36$ years old and $39.6 \pm 10.5$ years old among healthy non-menopausal women. All healthy volunteers did not have any significant medical conditions and reported no regular medications at enrolment.

\section{Vaginal microbiota}

The median vaginal $\mathrm{pH}$ in the SLE with and without UTI groups did not statistically differ $(5.3(5-5.5)$ vs 5.5 (4.4$5.5) ; \mathrm{p}=0.633)$. Of 52 patients, $15(28.8 \%)$ were discovered to have at least $50 \%$ of the sequences belonging to the Lactobacillus spp, and this proportion of patients was similar to that of the healthy volunteers (3 of 10, 30\%; figures 1 and 2). Thus, Lactobacillus spp were considered the predominant organisms in all participants. The proportions of Enterococcus spp, Peptostreptococcus spp and Veillonella dispar were higher in the SLE with UTI group than in the SLE without UTI group $(\mathrm{p}=0.038, \mathrm{p}=0.046$ and $\mathrm{p}=0.015$, respectively; table 1 ). Alpha diversity among all patients was determined via the Simpson_e index, and patients in the SLE with UTI group and SLE patients taking TMP-SMX prophylaxis had relatively low diversities compared with those of the controls $(\mathrm{p}=0.039$ and $\mathrm{p}=0.041$, respectively; figures 3 and 4 ). Patients' menstrual status did not affect the alpha diversity.

\section{Potential associated risk factors for UTI in this population}

TMP-SMX prophylaxis (OR: 16, 95\% CI 2.92 to 8.70 ; $\mathrm{p}=0.001$ ), elevated plasma C3 levels (OR: 12.75, 95\% CI 1.21 to 133.83; $\mathrm{p}=0.034$ ), and presence of Enterococcus spp (OR: 3.472, 95\% CI 1.04 to $11.55 ; \mathrm{p}=0.042)$ ), $V$. dispar (OR: 4.37, 95\% CI 1.29 to $14.73 ; \mathrm{p}=0.018$ ) and Peptostreptococcus spp (OR: $3.29,95 \%$ CI 1.00 to $10.79 ; \mathrm{p}=0.05$ ) in the vaginal microbiota were associated with an increased risk of UTI in the univariate analysis. In the multivariate logistic regression analysis, only three factors reached statistical significance: TMP-SMX prophylaxis (adjusted OR (AOR): 30.96, 95\% CI 3.63 to 264.11; $\mathrm{p}=0.002$ ), elevated plasma C3 levels (AOR: 35.33, 95\% CI 1.33 to 936.67; $\mathrm{p}=0.033$ ) and presence of $V$. dispar (AOR: 6.68, $95 \%$ CI 1.27 to $35.07 ; \mathrm{p}=0.025$; table 3 ).

\section{DISCUSSION}

Several factors affect UTI development in women. ${ }^{28}$ The unidirectional urine flow resulting from ureteric and bladder peristalsis helps reduce bacterial adhesion to epithelial cells. ${ }^{29}$ Another local mechanism is glycosaminoglycan secretion by bladder transitional cells, which acts as an antiadherence factor and traps bacteria. ${ }^{30}$ If the innate immune system fails to respond to the invading bacteria, the complement system and other complex mechanisms will occur next. C3 is one of the essential factors that promote opsonisation to speed up bacterial 
Co-morbidities

Table 1 Demographic and baseline characteristics

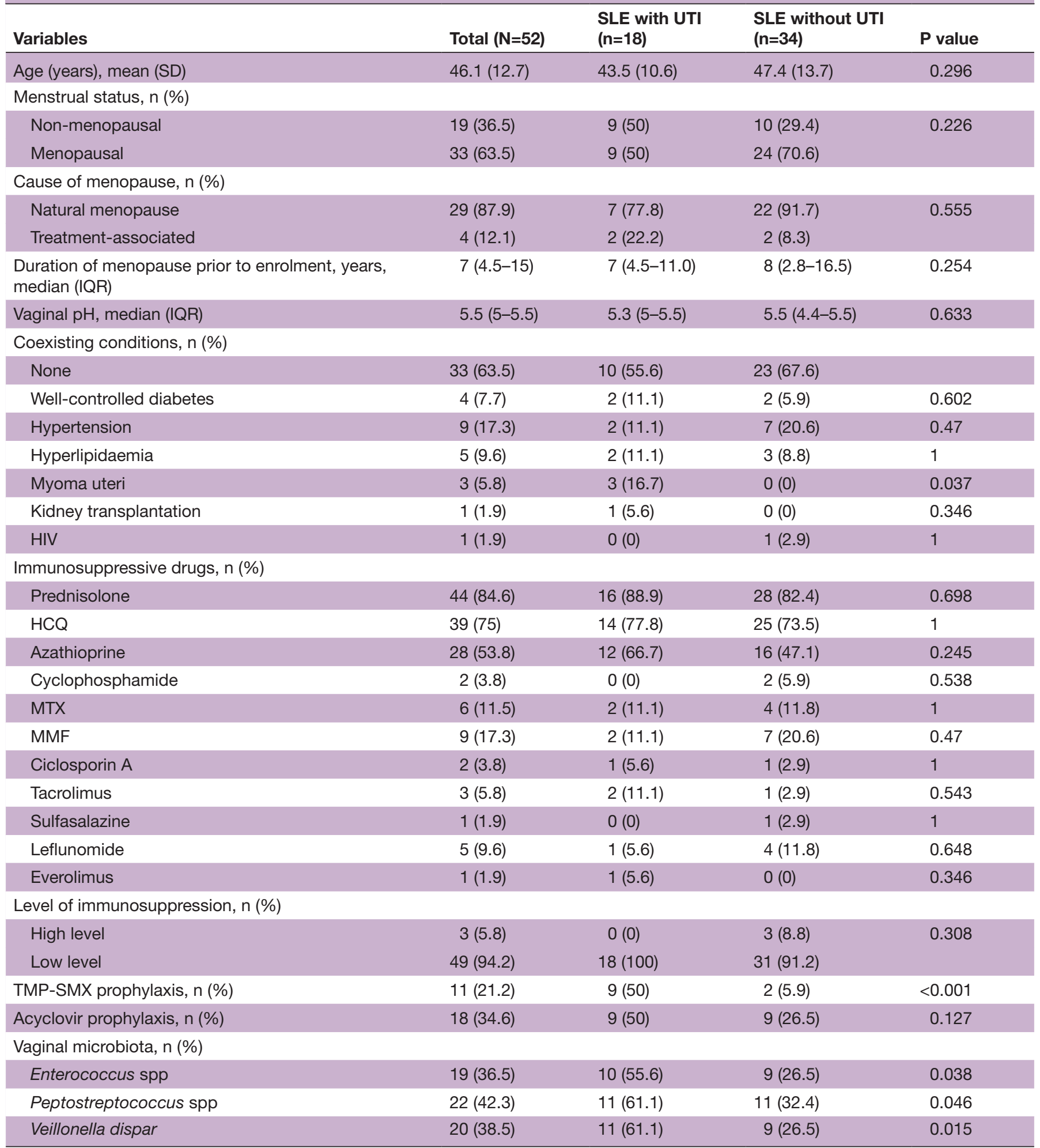

HCQ, hydroxychloroquine; MMF, mycophenolate mofetil; MTX, methotrexate; TMP-SMX, trimethoprim-sulfamethoxazole; UTI, urinary tract infection.

elimination. Deficient C3 may result in ineffective bacterial clearance, thus resulting in severe infection. ${ }^{30} 31$

Syukri et $a l^{31}$ noticed that serum C3 levels were significantly lower in patients with recurrent UTIs than in healthy younger women (mean $42.08 \mu \mathrm{g} / \mathrm{mL} \pm 1.20$ vs
$42.75 \mu \mathrm{g} / \mathrm{mL} \pm 0.71, \mathrm{p}=0.008)$. However, Springall $e t a l^{32}$ used a mouse model to demonstrate conflicting results in which C3 deficiency prevented an ascending renal infection. These authors postulated that the bacteria used C3 to enter renal epithelial cells, thus resulting in low C3 
Table 2 Three-month cumulative dose of immunosuppressive drugs

\begin{tabular}{llll}
\hline Immunosuppressive drugs & SLE with UTI $(\mathbf{n}=18)$ & SLE without UTI $(\mathbf{n}=\mathbf{3 4})$ & P value \\
\hline Prednisolone, mg/day, median (IQR) & $6.3(5.0-10.0)$ & $7.5(3.13-10.0)$ & 0.611 \\
HCQ, mg/day, mean \pm SD & $173.5 \pm 53.1$ & $173.5 \pm 53.8$ & 0.960 \\
Azathioprine, mg/day, mean \pm SD & $67.9 \pm 31.9$ & $58.7 \pm 24.0$ & 0.393 \\
MTX, mg/day, median (IQR) & $13.8(5.6-24.0)$ & $8.8(7.5-10.0)$ & 0.617 \\
MMF, mg/day, mean \pm SD & $1000 \pm 0$ & $1351.4 \pm 623.7$ & 0.667 \\
\hline
\end{tabular}

HCQ, hydroxychloroquine; MMF, mycophenolate mofetil; MTX, methotrexate; UTI, urinary tract infection.

levels. In our study, the mean serum C3 levels were higher in the SLE with UTI group than in the SLE without UTI group, possibly because C3 acts as an acute-phase protein and may be elevated during infectious episodes such as UTIs. ${ }^{33}$ However, determining the serum C3 level at one time point may not explain the entire clinical course but may be used to monitor dynamic changes in the complement. The vaginal microbiota is no less critical than the intact host immune responses. These microbes help maintain a healthy environment in the birth canal by hindering excessive growth of colonising uropathogens, thus reducing the risk of UTI. ${ }^{10}{ }^{34-37}$ The vaginal microbiota responds to uropathogens by competitive exclusion. Lactobacillus adheres to uroepithelial cells and produces lactic acid and other substances that alter the vaginal canal environment for excessive growth of specific pathogens. ${ }^{38-44}$

Prolonged antibiotic use may affect the vaginal microbiota, mostly reducing the microbial diversity. This effect triggers abnormal multiplication of the uropathogens, eventually altering the vaginal colonisation pattern. One animal study supported this theory: the researchers flushed cefadroxil into monkeys' vaginas and found predominant E. coli colonisation in 9 of 10 monkeys. Conversely, another group found no E. coli colonisation in the vaginal canals despite flushing them with P-fimbriated E. coli. ${ }^{45}{ }^{46}$ Consistently, in the current study, Enterococcus spp, Peptostreptococcus spp and $V$. dispar proportions were higher in the SLE with UTI group than in the SLE without UTI group $(\mathrm{p}=0.038, \mathrm{p}=0.046$ and $\mathrm{p}=0.015$, respectively). Additionally, the alpha diversity was low in the SLE with UTI group and in those who received TMP-SMX prophylaxis. Thus, prolonged use of a TMPSMX prophylaxis may affect the vaginal microbiota, but consequent UTI development requires additional clinical information to conclude.

Smith et $a l^{46}$ examined whether prior antimicrobial use influenced the subsequent relative risk of UTI in premenopausal women. These authors concluded that previous antibiotic use within 28 days as well as TMP-SMX were associated with an increased risk of UTI (relative risk: $5.83,95 \%$ CI 3.17 to 10.70 and relative risk: 4.56, 95\% CI 2.10 to 9.87 , respectively). Another retrospective study conducted at the University Student Health Center of the University of Florida between 2006 and 2014 revealed that TMP-SMX use as an initial agent in cystitis increased the risk of subsequent UTI events (AOR: 2.14, 95\% CI 1.23 to 4.09$).{ }^{47}$ This finding may be explained in that increasingly resistant bacteria caused the UTI, which could only be partially treated with TMP-SMX. Unknown effects on the vaginal microbiota may also cause recurrent infections. In our study, TMP-SMX prophylaxis was a potent associated risk factor of UTI (AOR: 30.96, 95\% CI 3.63 to 264.11; $\mathrm{p}=0.002$ ). Thus, prolonged use of TMP-SMX prophylaxis may cause unrecognised harm in patients with SLE.
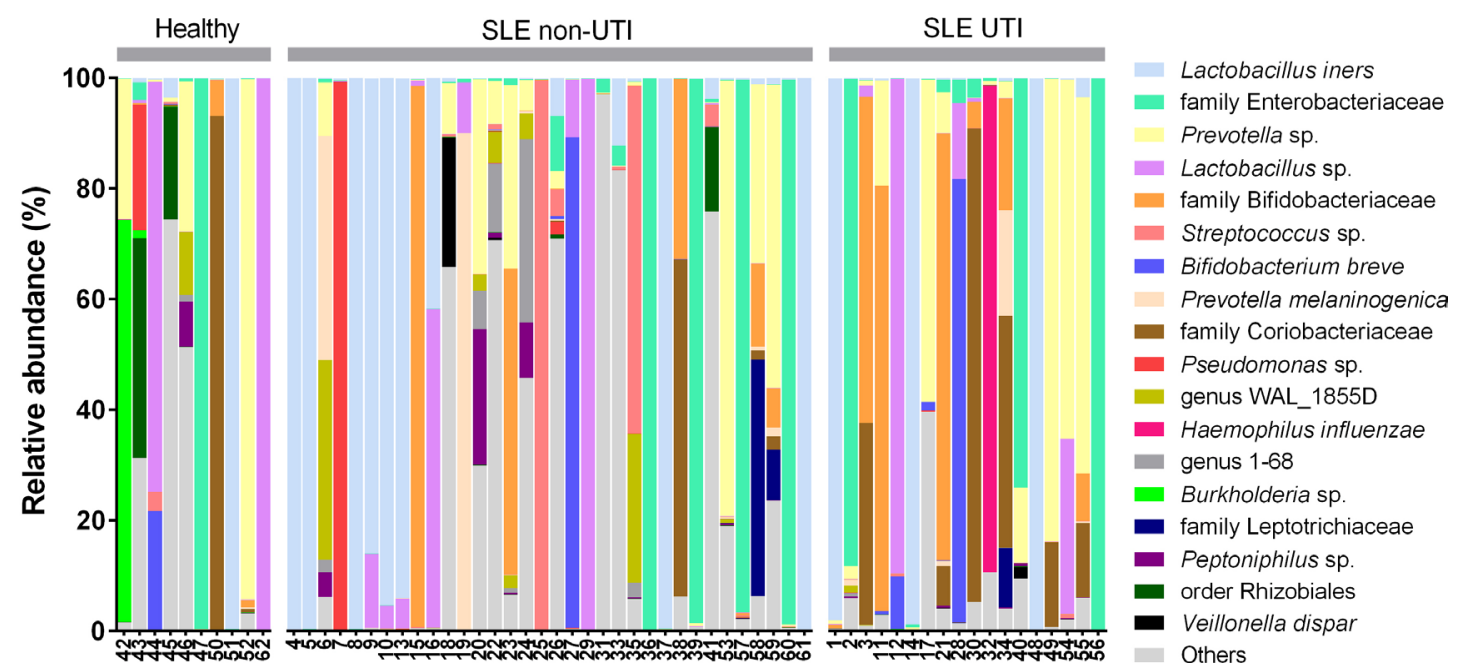

Figure 1 Relative abundance of taxa in vaginal samples from healthy controls, patients with SLE without UTI and patients with SLE with UTI. UTI, urinary tract infection. 


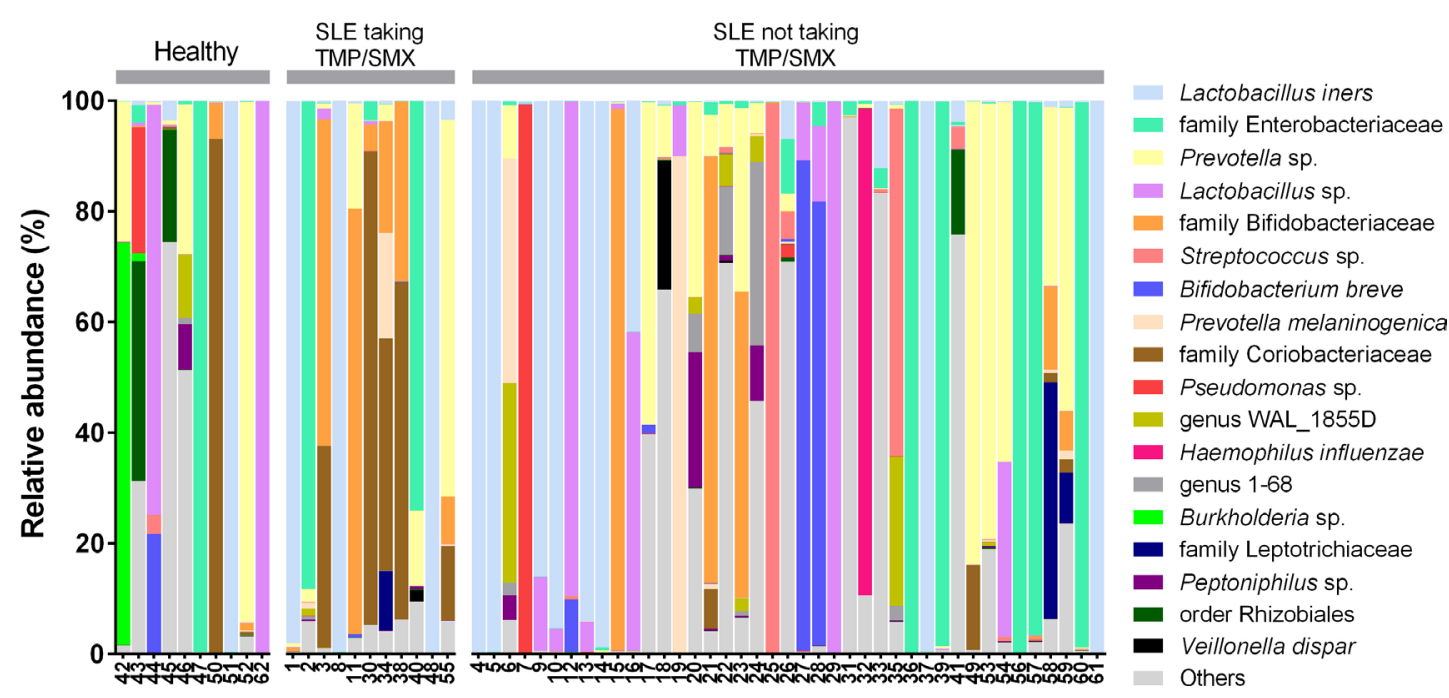

Figure 2 Relative abundance taxa bar plot of vaginal samples from healthy controls, patients with SLE taking TMP-SMX prophylaxis and patients with SLE not taking TMP-SMX prophylaxis. TMP-SMX, trimethoprim-sulfamethoxazole.

TMP-SMX is considered an effective treatment for uncomplicated UTIs; therefore, some clinicians acknowledge that patients may benefit from reduced risk of UTI, pneumocystis pneumonia and toxoplasmosis. However, one extensive clinical survey conducted at 28 hospitals across Thailand from 2000 to 2005, focusing on the susceptibility patterns of E. coli to TMP-SMX among non-critically ill individuals, demonstrated that $62.6 \%$ of the isolates were resistant to TMP-SMX. ${ }^{48}$ Therefore, routinely prescribing TMP-SMX to prevent UTI should be discouraged in Thailand.

TMP-SMX is indicated for opportunistic infection prophylaxis when CD4 counts are low or the patient is receiving high-dose corticosteroid therapy. ${ }^{49}$ However, to date, no consensus has been reached regarding when TMP-SMX should be provided to patients with SLE.

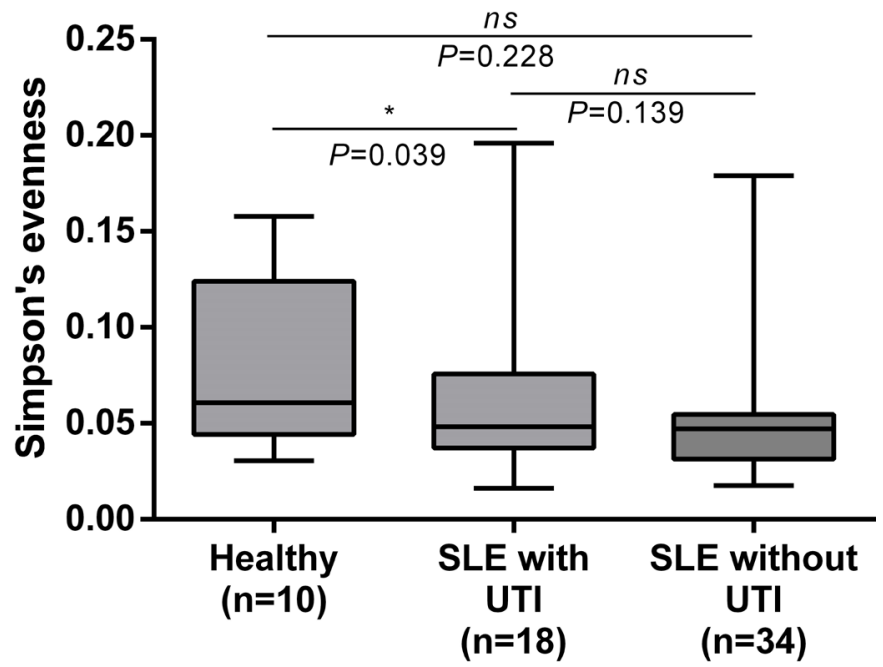

Figure 3 Distribution of alpha diversity within groups as measured by the Simpson_e index among healthy controls and patients with and without urinary tract infection (UTI). *lower alpha diversity among SLE with UTI and patients taking TMP-SMX prophylaxis compared with healthy controls.
Therefore, TMP-SMX for opportunistic infection prophylaxis as part of SLE treatment is solely individualised. Interestingly, most patients in our study were treated with only low-level immunosuppressive drugs, but a large proportion of them received TMP-SMX prophylaxis. Our data may reflect unnecessary treatment and provide evidence to support discontinuing TMP-SMX prophylaxis in patients with SLE who are receiving low-level immunosuppressive agents.

To further analyse whether TMP-SMX affects Lactobacillus proportions, Melkumyan et $a \tilde{l}^{00}$ conducted a clinical study assessing the sensitivity patterns of 123 vaginal Lactobacillus strains to a variety of antibiotic types and noticed that all strains were resistant to TMP-SMX. Hence,

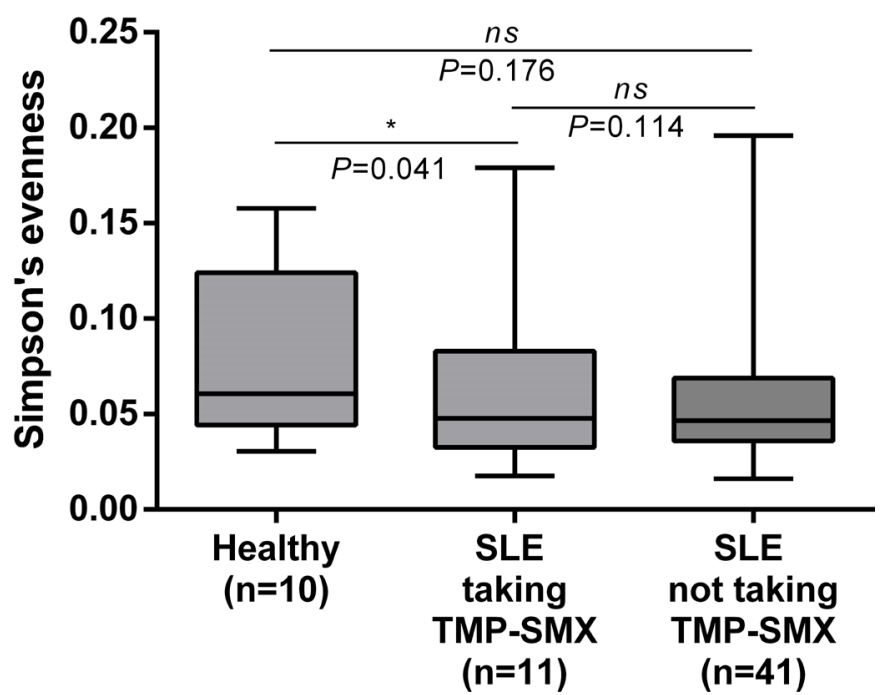

Figure 4 Distribution of alpha diversity within groups as measured by the Simpson_e index among healthy controls, patients with SLE taking TMP-SMX and patients with SLE not taking TMP-SMX prophylaxis. *lower alpha diversity among SLE with UTI and patients taking TMP-SMX prophylaxis compared with healthy controls. TMP-SMX, trimethoprimsulfamethoxazole. 
Table 3 Potential associated risk factors of urinary tract infection in female patients with SLE

\begin{tabular}{|c|c|c|c|c|}
\hline Variables & $\begin{array}{l}\text { Univariate analysis } \\
\text { OR }(95 \% \mathrm{Cl})\end{array}$ & $P$ value & $\begin{array}{l}\text { Multivariate analysis } \\
\text { OR }(95 \% \mathrm{Cl})\end{array}$ & $P$ value \\
\hline TMP-SMX prophylaxis & 16 (2.92 to 87.70$)$ & 0.001 & 30.96 (3.63 to 264.11$)$ & 0.002 \\
\hline \multicolumn{5}{|l|}{ Vaginal microbiota } \\
\hline Enterococcus spp & 3.472 (1.04 to 11.55$)$ & 0.042 & 5.53 (0.93 to 32.96$)$ & 0.060 \\
\hline
\end{tabular}

TMP-SMX, trimethoprim-sulfamethoxazole.

TMP-SMX should not directly affect the proportion of the main vaginal micro-organisms but rather affect the overall microbes in the vagina, causing reduced microbial diversity.

Our study had several limitations. First, the study population was small, and other essential risk factors of UTI may have been missed. Second, the nature of the crosssectional study did not allow comparing the vaginal microbiota patterns among individuals prior to and after TMP-SMX prophylaxis and prior to and after UTI events. Third, the results may not apply to active patients with SLE requiring a higher level of immunosuppressive therapy. A final limitation was that we lacked patients' histories of sexual activity. However, this study highlighted some significant findings as follows. First, vaginal microbiota diversity differs between patients with SLE with and without UTI. Second, unnecessary use of TMP-SMX prophylaxis may affect the alpha diversity of the vaginal microbiota.

In conclusion, UTI in patients with SLE remains a critical infectious complication that can increase patients' morbidity and mortality. In addition to comprehensively assessing the anatomical abnormalities of the genitourinary system, prophylactic agents must be carefully prescribed to help maintain a healthy and diverse vaginal microbiota and help prevent future UTI events. Further studies on a larger scale and with higher modified SLEDAI-2K scores are encouraged.

\section{Author affiliations}

${ }^{1}$ Division of Infectious Diseases, Department of Medicine, Faculty of Medicine Ramathibodi Hospital, Mahidol University, Bangkok, Thailand

${ }^{2}$ Division of Allergy, Immunology and Rheumatology, Department of Medicine, Faculty of Medicine Ramathibodi Hospital, Mahidol University, Bangkok, Thailand ${ }^{3}$ Division of Reproductive Health and Family Planning, Department of Obstetrics and Gynecology, Faculty of Medicine Ramathibodi Hospital, Mahidol University, Bangkok, Thailand

${ }^{4}$ Program in Bioinformatics and Computational Biology, Graduate School,

Chulalongkorn University, Bangkok, Thailand

${ }^{5}$ Research Unit of Systems Microbiology, Department of Biochemistry, Chulalongkorn University, Bangkok, Thailand

${ }^{6}$ Center of Excellence in Systems Biology (CUSB), Chulalongkorn University, Bangkok, Thailand

Acknowledgements We thank our colleagues at the Division of Allergy, Immunology and Rheumatology, Department of Medicine, Faculty of Medicine Ramathibodi Hospital, Mahidol University, and the staff at Ramathibodi Hospital for enrolling and caring for the participants and assisting in data collection.
Contributors PR and AJ conceived the study question and design and drafted the manuscript. AJ extracted the data and performed the statistical analysis. PR, PP, PN, JM, VS, PC and SP reviewed the analysis for accuracy. VS, PC and SP processed the samples and performed the DNA extraction, DNA sequencing and analysis. PR takes responsibility for the integrity of the data and the accuracy of the analysis. All authors had full access to the data (including statistical reports and tables) in the study and approved the final manuscript. PR was the principal investigator in this study and is also responsible for the overall content as the gurantor.

Funding This study was supported by the Faculty of Medicine Ramathibodi Hospital (RF_63040 and CF_61004) and Mahidol University (2563).

Disclaimer The funder had no role in the study design, data collection, analysis, decision to publish or manuscript preparation.

Competing interests None declared.

Patient consent for publication Consent obtained directly from patient(s). Ethics approval This study was conducted in accordance with the Declaration of Helsinki. Ethical approval was granted by the Research Ethics Committee of the Faculty of Medicine Ramathibodi Hospital, Mahidol University (approval no ID 0162-06). The committee approved the use of patient samples and data for publishing this study. All patients provided written informed consent.

Provenance and peer review Not commissioned; externally peer reviewed. Data availability statement Data are available in a public, open access repository.

Supplemental material This content has been supplied by the author(s). It has not been vetted by BMJ Publishing Group Limited (BMJ) and may not have been peer-reviewed. Any opinions or recommendations discussed are solely those of the author(s) and are not endorsed by BMJ. BMJ disclaims all liability and responsibility arising from any reliance placed on the content. Where the content includes any translated material, BMJ does not warrant the accuracy and reliability of the translations (including but not limited to local regulations, clinical guidelines, terminology, drug names and drug dosages), and is not responsible for any error and/or omissions arising from translation and adaptation or otherwise.

Open access This is an open access article distributed in accordance with the Creative Commons Attribution Non Commercial (CC BY-NC 4.0) license, which permits others to distribute, remix, adapt, build upon this work non-commercially, and license their derivative works on different terms, provided the original work is properly cited, appropriate credit is given, any changes made indicated, and the use is non-commercial. See: http://creativecommons.org/licenses/by-nc/4.0/.

\section{ORCID iDs}

Porpon Rotjanapan http://orcid.org/0000-0003-0966-2708

Pintip Ngamjanyaporn http://orcid.org/0000-0001-7948-1748

\section{REFERENCES}

1 La Paglia GMC, Leone MC, Lepri G, et al. One year in review 2017: systemic lupus erythematosus. Clin Exp Rheumatol 2017;35:551-61.

2 Esposito S, Bosis S, Semino M, et al. Infections and systemic lupus erythematosus. Eur J Clin Microbiol Infect Dis 2014;33:1467-75.

3 Hidalgo-Tenorio C, Jiménez-Alonso J, de Dios Luna J, et al. Urinary tract infections and lupus erythematosus. Ann Rheum Dis 2004;63:431-7.

4 Foxman B. The epidemiology of urinary tract infection. Nat Rev Urol 2010;7:653-60. 
5 Minardi D, d'Anzeo G, Cantoro D, et al. Urinary tract infections in women: etiology and treatment options. Int J Gen Med 2011;4:333.

6 John AS, Mboto Cl, Agbo B. A review on the prevalence and predisposing factors responsible for urinary tract infection among adults. Euro J Exp Bio 2016;6:7-11.

7 Lindblad A, Persson K, Demirel I. II-1Ra is part of the inflammasome-regulated immune response in bladder epithelial cells and influences colonization of uropathogenic E. coli. Cytokine 2019;123:154772-9.

8 Kirjavainen PV, Pautler S, Baroja ML, et al. Abnormal immunological profile and vaginal microbiota in women prone to urinary tract infections. Clin Vaccine Immunol 2009;16:29-36.

9 Ravel J, Gajer P, Abdo Z, et al. Vaginal microbiome of reproductiveage women. Proc Natl Acad Sci U S A 2011;108:4680-7.

10 Stapleton AE. The vaginal microbiota and urinary tract infection. Microbiol Spectr 2016;4

11 O'Hanlon DE, Moench TR, Cone RA. Vaginal pH and microbicida lactic acid when lactobacilli dominate the microbiota. PLoS One 2013;8:e80074.

12 Hawes SE, Hillier SL, Benedetti J, et al. Hydrogen peroxideproducing lactobacilli and acquisition of vaginal infections. J Infect Dis 1996;174:1058-63.

13 Martin HL, Richardson BA, Nyange PM, et al. Vaginal lactobacilli, microbial flora, and risk of human immunodeficiency virus type 1 and sexually transmitted disease acquisition. $J$ Infect Dis 1999;180:1863-8.

14 Nielsen KL, Dynesen P, Larsen P, et al. Faecal Escherichia coli from patients with E. coli urinary tract infection and healthy controls who have never had a urinary tract infection. $J$ Med Microbiol 2014;63:582-9.

15 Nicolle LE, Bradley S, Colgan R, et al. Infectious diseases Society of America guidelines for the diagnosis and treatment of asymptomatic bacteriuria in adults. Clin Infect Dis 2005;40:643-54.

16 Gupta K, Hooton TM, Naber KG, et al. International clinical practice guidelines for the treatment of acute uncomplicated cystitis and pyelonephritis in women: a 2010 update by the infectious diseases Society of America and the European Society for microbiology and infectious diseases. Clin Infect Dis 2011;52:e103-20.

17 America IDSo.. IDSA clinical practice guideline for vaccination of the immunocompromised host, 2013.

18 Yong PF, D'Cruz DP, D'Cruz DP. Mycophenolate mofetil in the treatment of lupus nephritis. Biologics 2008;2:297.

19 Gladman DD, Ibañez D, Urowitz MB. Systemic lupus erythematosus disease activity index 2000. J Rheumatol 2002;29:288-91

20 Mikdashi J, Nived O. Measuring disease activity in adults with systemic lupus erythematosus: the challenges of administrative burden and responsiveness to patient concerns in clinical research. Arthritis Res Ther 2015;17:183.

21 Walters KE, Martiny JBH, Kendra E. Alpha-, beta-, and gamma-diversity of bacteria varies across habitats. PLOS One 2020;15:e0233872-17.

22 Wagner BD, Grunwald GK, Zerbe GO, et al. On the use of diversity measures in longitudinal sequencing studies of microbial communities. Front Microbiol 2018;9:1-11.

23 Wu L, Wen C, Qin Y, et al. Phasing amplicon sequencing on Illumina Miseq for robust environmental microbial community analysis. BMC Microbiol 2015;15:125.

24 Bolyen E, Rideout JR, Dillon MR, et al. Reproducible, interactive, scalable and extensible microbiome data science using QIIME 2. Nat Biotechnol 2019;37:852-7.

25 Rognes T, Flouri T, Nichols B, et al. VSEARCH: a versatile open source tool for metagenomics. PeerJ 2016;4:e2584.

26 Edgar RC, Haas BJ, Clemente JC, et al. UCHIME improves sensitivity and speed of chimera detection. Bioinformatics 2011;27:2194-200.

27 DeSantis TZ, Hugenholtz P, Larsen N, et al. Greengenes, a chimerachecked 16S rRNA gene database and workbench compatible with ARB. Appl Environ Microbiol 2006;72:5069-72.
28 Scholes D, Hooton TM, Roberts PL, et al. Risk factors for recurrent urinary tract infection in young women. $J$ Infect Dis 2000;182:1177-82

29 Cox CE, Hinman F. Experiments with induced bacteriuria, vesical emptying and bacterial growth on the mechanism of bladder defense to infection. J Urol 1961;86:739-48.

30 Springall T, Sheerin NS, Sacks SH. Breaching host defenses in the urinary tract. Hong Kong J Nephrol 2002;4:13-21.

31 Syukri M, Imran I, Harapan H, et al. Comparison of serum C3 complement levels between young women with recurrent urinary tract infection and healthy women. Alexandria J Med 2015;51:35-9.

32 Springall T, Sheerin NS, Abe K, et al. Epithelial secretion of C3 promotes colonization of the upper urinary tract by Escherichia coli. Nat Med 2001;7:801-6.

33 Alava MA, González-Ramón N, Heegaard P, et al. Pig-MAP, porcine acute phase proteins and standardisation of assays in Europe. Comp Haematol Int 1997;7:208-13.

34 Czaja CA, Stamm WE, Stapleton AE, et al. Prospective cohort study of microbial and inflammatory events immediately preceding Escherichia coli recurrent urinary tract infection in women. $J$ Infect Dis 2009:200:528-36.

35 Russo TA, Stapleton A, Wenderoth S, et al. Chromosomal restriction fragment length polymorphism analysis of Escherichia coli strains causing recurrent urinary tract infections in young women. $J$ Infect Dis 1995;172:440-5.

36 Beerepoot M, Geerlings S. Non-Antibiotic prophylaxis for urinary tract infections. Pathogens 2016;5:36

37 Mulvey MA, Klumpp DJ, Stapleton AE. Urinary tract infections: molecular pathogenesis and clinical management. John Wiley \& Sons, 2017

38 Boris $\mathrm{S}$, Barbés $\mathrm{C}$. Role played by lactobacilli in controlling the population of vaginal pathogens. Microbes Infect 2000;2:543-6.

39 Herthelius M, Gorbach SL, Möllby R, et al. Elimination of vaginal colonization with Escherichia coli by administration of Indigenous flora. Infect Immun 1989;57:2447-51.

40 McGroarty JA, Reid G. Detection of a Lactobacillus substance that inhibits Escherichia coli. Can J Microbiol 1988;34:974-8.

41 McGroarty JA, Tomeczek L, Pond DG, et al. Hydrogen peroxide production by Lactobacillus species: correlation with susceptibility to the spermicidal compound nonoxynol-9. J Infect Dis 1992;165:1142-4.

42 Osset J, Bartolomé RM, García E, et al. Assessment of the capacity of Lactobacillus to inhibit the growth of uropathogens and block their adhesion to vaginal epithelial cells. J Infect Dis 2001;183:485-91.

43 Reid G, Cook RL, Bruce AW. Examination of strains of lactobacilli for properties that may influence bacterial interference in the urinary tract. J Urol 1987;138:330-5.

44 Reid G, Heinemann C, Velraeds M. Biosurfactants produced by Lactobacillus. In: Methods in enzymology. . Elsevier, 1999: 310. 426-33.

45 Winberg J, Gezelius L, Guldevall L, et al. Cephadroxil promotes vaginal colonization with Escherichia coli. Infection 1993;21:201-5.

46 Smith HS, Hughes JP, Hooton TM, et al. Antecedent antimicrobial use increases the risk of uncomplicated cystitis in young women. Clin Infect Dis 1997;25:63-8.

47 Rich SN, Klann EM, Almond CR, et al. Associations between antibiotic prescriptions and recurrent urinary tract infections in female college students. Epidemiol Infect 2019;147:e119.

48 Polwichai P, Dejsirilert S, Panpetch S. Antimicrobial resistance of Escherichia coli isolated from urine in Thailand from 2000 to 2005. $J$ Med Assoc Thai 2011;92:S59-67.

49 Maertens J, Cesaro S, Maschmeyer G, et al. ECIL guidelines for preventing Pneumocystis jirovecii pneumonia in patients with haematological malignancies and stem cell transplant recipients. $J$ Antimicrob Chemother 2016;71:2397-404.

50 Melkumyan AR, Priputnevich TV, Ankirskaya AS, et al. Effects of antibiotic treatment on the Lactobacillus composition of vaginal microbiota. Bull Exp Biol Med 2015;158:766-8. 